\title{
ELECTRIC DIPOLE CENTRES AND COLOUR CENTRES IN NATURAL SODALITE
}

\author{
W. E. VAN DEN BROM, J. KERSSEN and J. VOLGER \\ Fysisch Laboratorium der Rijksuniversiteit Utrecht, Afdeling Vaste Stof Fysica, \\ Utrecht, The Netherlands
}

Received 2 August 1974

\begin{abstract}
Synopsis
Experiments on an electric dipole centre, exhibiting multiple relaxation, in the natural mineral sodalite, are described. The concentration of the dipole centres is reduced upon $\mathrm{X}$ irradiation, whereas simultaneously colour centres and paramagnetic centres arise. Thermal bleaching restores the original concentrations. The interrelationships between these centres are established.

A model is proposed in which the dipole centre is ascribed to an interstitial monovalent metal ion (say a $\mathrm{Na}^{+}$ion) acting as a charge compensator for an $\mathrm{Al}^{3+}$ ion, substituted for a $\mathrm{Si}^{4+}$ ion. This $\mathrm{Al}-\mathrm{Na}$ complex may be destroyed by $\mathrm{X}$ rays, yielding an electron trapped at the sodium ion and a hole trapped at a nonbridging oxygen ion, adjacent to the $\mathrm{Al}^{3+}$ ion, to which both the optical and paramagnetic properties of the $\mathrm{X}$ ray induced centres are attributed.
\end{abstract}

1. Introduction. Sodalite is an alumino-silicate with chemical composition $\mathrm{Na}_{4}\left(\mathrm{AlSiO}_{4}\right)_{3} \mathrm{Cl}$. In the past it has received some attention in connection with the luminescence, exhibited by one of the varieties (Hackmanite) of the mineral ${ }^{1,2}$ ). More recently there has been a considerable interest for the photochromic and cathodochromic properties, which sodalite exhibits in certain circumstances, in connection with its possible application as screen material in storage display tubes ${ }^{\mathbf{3}, \mathbf{4}}$ ).

The structure of sodalite can be visualized as follows. $\mathrm{SiO}_{4}$ and $\mathrm{AlO}_{4}$ tetrahedra build up a framework in such a way that cages are formed with the form of cubo-octahedra, resulting in a cubic crystal symmetry ${ }^{5}$ ). The vertices of the cubo-octahedron are occupied alternatingly by silicon and aluminium ions. Any oxygen ion is shared by two tetrahedra. Every cage contains a $\mathrm{Cl}^{-}$ion, surrounded by four $\mathrm{Na}^{+}$ions.

The photochromic properties are closely related to the presence of $\mathrm{F}$ centres. As appeared from ESR experiments ${ }^{6}$ ), these are chlorine vacancies in which electrons are trapped. The presence of sulphur as a contamination 
seems to be desirable for an optimal photochromic behaviour ${ }^{3}$ ), and also plays an important part in the explanation of the above mentioned luminescence ${ }^{2,7}$ ). ESR experiments ${ }^{6,8}$ ) reveal that many sulphur complexes may occur, presumably within the cages.

In view of the complicated structure one would indeed expect many kinds of defects to be present. We are especially interested in the presence* and behaviour of electric dipole centres which may reveal their existence by the occurrence of dielectric relaxation phenomena. In the following sections experiments will be described, concerning such centres themselves as well as their relation to other centres.

2. Experimental procedures. Single crystals of sodalite are rare, especially when it concerns crystals of dimensions suitable for our dielectric experiments (say an area of $0.5-1 \mathrm{~cm}^{2}$ and a thickness of $0.2 \mathrm{~mm}$ ). Most of the samples investigated in the above mentioned literature were prepared by hydrothermal methods. Those methods usually produce crystalline powders. In order to avoid any complications connected with the use of powders in the dielectric investigations we preferred to use in our experiments the natural blue coloured mineral, although it is not monocrystalline. Only a few years ago Bye and White ${ }^{10}$ ) have succeeded in preparing single crystals with dimensions more suitable for our use. A number of dielectric experiments was performed on such a samplet.

The blue colour of the mineral disappears when the sample is heated to about $450^{\circ} \mathrm{C}$, whereas the same colour is restored by $\mathrm{X}$ irradiation ${ }^{1}$ ). We employed a $50 \mathrm{kV}, 32 \mathrm{~mA}$ tube with a tungsten anode, supplying a flux of $0.3 \mathrm{Mrad} /$ hour. The irradiations were performed at room temperature. It appeared that $X$ rays may strongly influence the dielectric, optical and paramagnetic properties of sodalite.

In the dielectric experiments the loss angle $\tan \delta$ was determined. The measurements were carried out at frequencies up to about $3 \mathrm{MHz}$, using two Schering type bridges: GR 716C and $716 \mathrm{CS}$. The accuracy in $\tan \delta$ was about $2 \times 10^{-4}$. Electrodes on the sample were obtained by applying silver paint. Between $20 \mathrm{~K}$ and $300 \mathrm{~K}$ a small Joule-Thomson hydrogen liquefier cryostat was used, and below $20 \mathrm{~K}$ a simple helium cryostat. Temperatures were measured by means of a copper resistor and a germanium resistor, respectively.

\footnotetext{
* Recently, it has been pointed out by R. Casanova Alig ${ }^{9}$ ) that vacancy pairs, possessing electric dipole moments, may exist in the cages of sensitized sodalite. However, these do not seem to account for our experimental results.

†We are much indebted to Dr. K. L. Bye for putting a sample at our disposal.
} 
The optical absorption was measured with a double-beam spectrophotometer (Perkin Elmer EPS-3T), covering the range between $220 \mathrm{~nm}$ and $2600 \mathrm{~nm}$. The absorption was examined at room temperature. Some additional infrared absorption measurements were also performed (Hitachi EPI-G3 and Grubb Parsons DM4).

The ESR experiments were carried out with a laboratory-built X-band spectrometer. The samples were mounted in a TE 011 cavity inside a cryostat, suitable for measurements between about $10 \mathrm{~K}$ and room temperature. The magnetic field strength was measured with an AEG NMR Gaussmeter and the microwave frequency with a HP 5257A transfer oscillator.

3. Dielectric relaxation experiments. A medium with electric dipoles relaxing under the influence of an applied electric field can be described by a complex dielectric constant $\epsilon_{1}+\mathrm{i} \epsilon_{2}$. If the Debye model is applicable, $\epsilon_{1}$ and $\epsilon_{2}$ are related by the well-known Debye equations ${ }^{11}$ ):

$$
\begin{aligned}
& \epsilon_{1}=\epsilon_{\infty}+\frac{\epsilon_{\mathrm{s}}-\epsilon_{\infty}}{1+\omega^{2} \tau^{2}}, \\
& \epsilon_{2}=\left(\epsilon_{\mathrm{s}}-\epsilon_{\infty}\right) \frac{\omega \tau}{1+\omega^{2} \tau^{2}},
\end{aligned}
$$

in which $\omega$ denotes the frequency of the applied field and $\tau$ the relaxation time of the dipoles. $\epsilon_{\mathrm{s}}$ and $\epsilon_{\infty}$ are the values of $\epsilon_{1}$ for frequencies $\omega$ if $\omega \ll \tau^{-1}$ and $\omega \gg \tau^{-1}$, respectively. The quantity measured in the relaxation loss experiments is the loss angle, given by

$$
\tan \delta=\frac{\epsilon_{2}}{\epsilon_{1}}=\frac{C}{T} \frac{\omega \tau}{1+\omega^{2} \tau^{2}},
$$

where $T$ is the temperature and $C / T=\left(\epsilon_{\mathrm{s}}-\epsilon_{\infty}\right) / \epsilon_{\mathrm{s}}$. Equation (2) holds if $\epsilon_{\mathrm{s}}-\epsilon_{\infty} \ll 1$. $C$ is proportional to the dipole concentration and the square of the dipole moment.

Generally $\tau$ depends on the temperature; in many cases its dependence is given by

$$
\tau=\tau_{0} \exp (U / k T)
$$

The activation energy $U$ is usually interpreted as the height of a potential barrier separating two different orientations of the dipole involved. 
3.1. Description of the observed relaxation processes. We investigated five sodalite samples, all of them exhibiting relaxation losses below room temperature. The relaxation time $\tau$, characteristic for the relaxation process, can be obtained from the condition that the loss reaches a maximum: $\omega \tau=1$ [eq.(2)] . In fig. 1 the experimentally determined $\tau$ values have been plotted versus $T^{-1}$. This figure shows that in the frequency range at our disposal two relaxation processes are present, with relaxation times that indeed follow eq.(3). Labelling the processes around $90 \mathrm{~K}$ and $35 \mathrm{~K}$ process I and II, respectively, we find for $\tau_{0}$ and $U$ :

$$
\begin{aligned}
& \text { process I: } \tau_{0}=(1.7 \pm 0.2) \times 10^{-13} \mathrm{~s} ; U=150 \pm 5 \mathrm{meV} \\
& \text { process II: } \tau_{0}=(5 \pm 0.5) \times 10^{-13} \mathrm{~s} ; U=55 \pm 5 \mathrm{meV}
\end{aligned}
$$

These values were found in all the investigated samples.

However, the magnitude of the loss, i.e., the maximum of $T \tan \delta$, varies strongly and, in addition, depends on the condition of the material. It appears that the disappearance of the blue colour of the mineral by thermal bleaching at $450^{\circ} \mathrm{C}$ is accompanied by an increase of the loss. On the other hand, $\mathrm{X}$ irradiation, which restores the colour, diminishes the loss. These remarks apply to both process I and process II. Examining, however, the ratio of the maximum value of the losses for both processes, i.e. the ratio

$$
\eta^{2}=\left(T \tan \delta_{\max }\right)_{\mathrm{I}} /\left(T \tan \delta_{\max }\right)_{\mathrm{II}}
$$

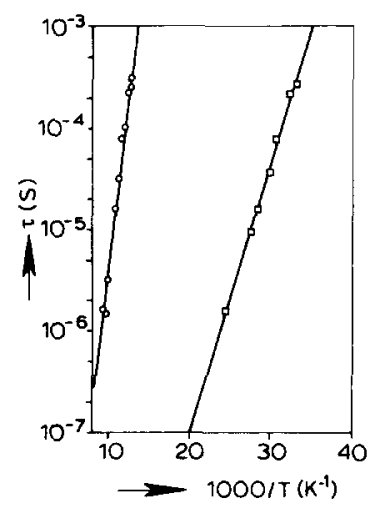

Fig. 1. Temperature dependence of the experimental relaxation times for the sample E-2. Other samples show essentially the same plot. Relaxation processes I and II are denoted by $O$ and $\square$, respectively. 
we find that $\eta^{2}$ does not change significantly by the bleaching or the irradiation and, moreover, does not depend on the choice of the sample. The value of $\eta$ gives some information about the ratio of the dipole moments involved in both relaxation processes if the dipole concentration in both cases is the same. From our experiments we deduced $\eta=2.7 \pm 0.3$.

The experimental loss curves are in general more or less broadened with respect to the ideal Debye curve [ the curve as given by eq.(3)]. In fig. 2 an example is given. By way of comparison we included in the figure also the corresponding ideal curve. The curves belonging to process I are scarely broadened whereas those belonging to process II are strongly broadened, usually in an asymmetric way without any obvious temperature dependence. The broadening, which can be explained by the existence of a certain distribution of relaxation times instead of a single one [as assumed in eq.(3)], can be analysed in many cases by the method of Kirkwood and Fuoss ${ }^{11}$ ). According to this method eq. (3) is replaced quite formally by

$$
\begin{aligned}
\epsilon_{2}(\omega \tau) & =2 \epsilon_{2 \max } \frac{(\omega \tau)^{\alpha}}{1+(\omega \tau)^{2 \alpha}} \\
& =\epsilon_{2 \max } \operatorname{sech}(\alpha \log \omega \tau),
\end{aligned}
$$

so that $\alpha \log \omega \tau=\operatorname{arccosh}\left[\epsilon_{2 \max } / \epsilon_{2}(\omega \tau)\right]$.

By plotting the right-hand side of this equation as a function of $\log \omega$, or $\log \tau$, the value of $\alpha$ can be determined. (For an ideal Debye curve $\alpha=1$, for the other cases $\alpha<1$.) The description of Fuoss and Kirkwood can be adapted simply to the case of the asymmetrical broadening by taking two different values of $\alpha: \alpha=\alpha_{+}$for $\omega \tau \geqslant 1$ and $\alpha=\alpha_{-}$for $\omega \tau<1$. The ratio

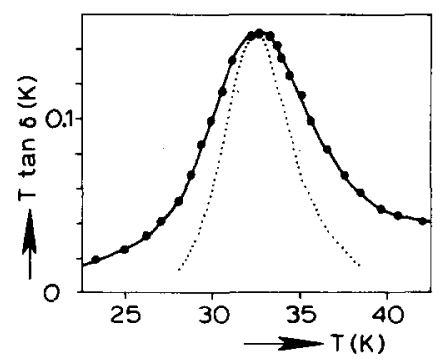

Fig. 2. An experimental loss curve, characteristic for process II; measured at $2 \mathrm{kHz}$ (sample F-1, thermally bleached). The broken curve represents the unbroadened Debye curve. 
$\alpha_{+} / \alpha_{-}$is then a measure of the asymmetry. In fact this approach is a special case of the Fuoss-Kirkwood method as modified for asymmetrical loss curves by De Vos and Volger ${ }^{12}$ ).

As a consequence of the broadening the value of $T \tan \delta_{\max }$ decreases by a certain factor $G$. From the Kramers-Kronig relations it follows for this correction factor ${ }^{12}$ ):

$$
G=\alpha^{-1}=\frac{1}{2}\left(\alpha_{+}^{-1}+\alpha_{-}^{-1}\right) .
$$

Experimentally we found:

$$
G=1.05 \pm 0.05 \quad \text { (process I) }
$$

and

$$
G=1.55 \pm 0.15 \quad \text { (process II). }
$$

In order to find the value of $C$ in eq.(2) we have to correct the maximum values of the losses by these factors. As a consequence the ratio $\eta$, as corrected for the broadening of the curves, becomes:

$$
\eta=2.2 \pm 0.2
$$

The ratio $\alpha_{+} / \alpha_{-}$does not differ much from unity for process I, whereas it takes on values between 0.9 and 1.7 for process II. The fact that the broadening is more pronounced for the low-temperature relaxation process than for the high-temperature one is not unusual ${ }^{11}$ ).

So far the results concern experiments performed on the natural mineral. We also measured the loss of the synthetically grown sample P. This simple exhibited the process I type of relaxation losses. We could not detect losses around $35 \mathrm{~K}$ (process II). In fact this is to be expected because, if the process II were present, its magnitude would be smaller relative to process I by a factor $\eta^{2} \approx 8$. Since the magnitude of $T \tan \delta_{\max }$ in process I amounts to only $0.07 \mathrm{~K}$, it therefore would fall beyond the limit of detection. In view of these considerations we believe the same dipole centres to be responsible for the losses in both the natural mineral and the synthetically grown sample.

3.2. The influence of $X$ irradiation. For two samples we investigated the decrease of the loss under the influence of $\mathrm{X}$ irradiation. For that purpose the samples were first thermally bleached: after determination of the loss they were irradiated and the loss was measured again. The 
following irradiations took place without preceding bleaching, i.e. we assumed the effect of irradiation to be additive. This assumption was confirmed in a separate experiment.

Owing to the already mentioned fact that the ratio $\eta^{2}$ is independent of the irradiation time we could confine ourselves to the determination of the loss in process I.

The samples investigated (E-2 and F-1) exhibited a fast initial decrease of the loss (stage 1), followed by a slow decrease (stage 2). Finally the loss reached a constant value, differing from zero (fig. 3 ). In both stages the decay is exponential with a characteristic rate $\gamma$ according to

$$
T \tan \delta_{\max } \propto \exp (-\gamma t) .
$$

The experimental values of $\gamma$ are:

$$
\begin{aligned}
& \text { for E-2: } \gamma_{1}=0.345 \text { hour }^{-1} ; \gamma_{2}=0.084 \text { hour }^{-1}, \\
& \text { for F-1: } \gamma_{1}=0.095 \text { hour }^{-1} ; \gamma_{2}=0.024 \text { hour }^{-1}
\end{aligned}
$$

Note that these values hold for the particular X-ray intensity we used (0.3 Mrad/h).

Although the loss decreases qualitatively in the same way for both samples, there is a great quantitative difference between the two samples.

4. Optical experiments. As mentioned above, the dielectric loss, being maximal for the bleached material, decreases under the influence of irradiation. This decrease is accompanied by an increase of the blue colour. One may wonder whether there exists a relation between the dipole centre and the colour centre responsible for the blue colour. We

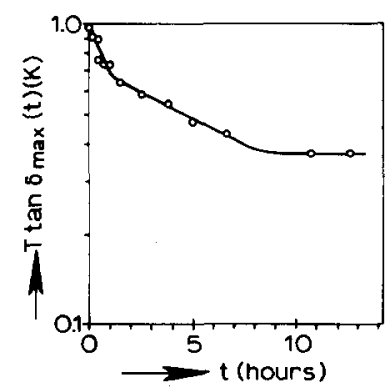

Fig. 3. Decrease of the loss (relaxation process I) as a function of the irradiation time $t$ (sample E-2). 
therefore studied the optical properties, especially the optical absorption in the visible region. The experiments were performed at room temperature.

4.1. The optical absorption at $610 \mathrm{~nm}$. All the samples showed a broad absorption band around $610 \mathrm{~nm}$, being equivalent to a photon energy of $2.04 \mathrm{eV}$. We calculated the absorption coefficient $\alpha$ from the transmission spectrum by means of the relation

$$
\alpha=(1 / d) \log \left(T_{0} / T_{1}\right)
$$

in which $d$ is the thickness of the sample, $T_{1}$ the measured transmission and $T_{0}$ the transmission when the absorption band is absent. An example of the dependence of $\alpha$ on the photon energy $\epsilon$ is given in fig. 4 .

For a number of samples and irradiation times we determined the ratio

$$
a_{\mathrm{S}}=M_{0} / \alpha_{m} H,
$$

where $M_{0}=\int \alpha(\epsilon) \mathrm{d} \epsilon, \alpha_{\mathrm{m}}$ is the maximum value of $\alpha$ and $H$ the width of the band at half-maximum. For a gaussian and a lorentzian line the constant $a_{\mathrm{S}}$ (sometimes called Smakula's constant ${ }^{13}$ )) is 1.06 and 1.57 , respectively. The analysis yields:

$$
H=0.71 \pm 0.02 \mathrm{eV} \quad \text { and } \quad a_{\mathrm{S}}=1.02 \pm 0.02 \text {. }
$$

Neither the position nor the width of the band depends significantly on the irradiation time. The same applies to Smakula's constant. Although a gaussian fit is not very successful, the experimental value of $a_{\mathrm{S}}$ and the theoretical one for a gaussian line are almost equal.

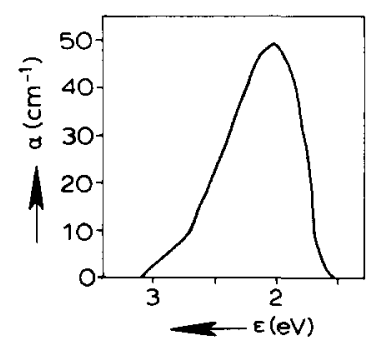

Fig. 4. The optical absorption spectrum of sample F-2 after irradiation for 3 hours. 
Denoting by $\epsilon_{\mathrm{r}}$ and $\epsilon_{\mathrm{v}}$ the value of $\epsilon$ for which $\alpha=\frac{1}{2} \alpha_{\mathrm{m}}$ with $\epsilon_{\mathrm{r}}<\epsilon_{\mathrm{m}}<\epsilon_{\mathrm{v}}$ one may describe the asymmetry of the line by the ratio $\left(\epsilon_{\mathrm{v}}-\epsilon_{\mathrm{m}}\right) /\left(\epsilon_{\mathrm{m}}-\epsilon_{\mathrm{r}}\right)$. For all the investigated samples we found for this ratio 1.3 , independent of the irradiation time.

In the case of electric dipole transitions the theoretical expression for the absorption coefficient is given by ${ }^{14}$ )

$$
\alpha(\epsilon)=\frac{\pi e^{2}}{m c} \frac{1}{n} \kappa^{2} f N_{\mathrm{c}} g(\epsilon)
$$

in which $g(\epsilon)$ is the normalized line shape, $f$ the oscillator strength of the transition, $N_{\mathrm{c}}$ the colour centre concentration, $\kappa$ a factor giving the ratio between the local electric field and the field in vacuo, and $n$ is the index of refraction. With the help of eq.(9) this expression may be rewritten as

$$
f N_{\mathrm{c}}=\frac{n}{\kappa^{2}} \frac{m c}{\pi e^{2}} a_{\mathrm{s}} \alpha_{\mathrm{m}} H .
$$

If we assume that in the observed absorption only one kind of centre is involved, we find by substitution into eq.(11) for $\alpha_{\mathrm{m}}$ and $H$ the values given in (10), taking $n=1.485^{15}$ ), and applying a Lorentz field correction $\kappa=\left(n^{2}+2\right) / 3$ :

$$
f N_{\mathrm{c}}=5.0 \times 10^{15} \alpha_{\mathrm{m}}\left(\mathrm{cm}^{-3}\right)
$$

with $\alpha_{\mathrm{m}}$ in $\mathrm{cm}^{-1}$.

4.2. The growth curve. The way in which $\alpha_{\mathrm{m}}$ increases under the influence of the $X$ irradiation was investigated for three samples. We started with bleached samples and assumed that the effect of irradiation was additive. This was checked in a separate experiment: the processes of colouring and bleaching are reversible.

The experimental growth curves behave according to

$$
\alpha_{\mathrm{m}}(t)=\alpha_{\mathrm{s}}\left[1-\exp \left(-\gamma_{\mathrm{c}} t\right)\right]
$$

where $\alpha_{\mathrm{s}}$ is the saturation value of the absorption coefficient. Fitting this expression to the experimental points yields

$$
\gamma_{c}=0.30,0.45 \text { and } 0.45 \text { hour }^{-1}
$$

for F-2, E-2 and B-5, respectively. 
Equation (13) suggests that the process of colouring could be described by a model in which the irradiation converts "latent" centres into colour centres. If the concentration $n(t)$ of latent centres decreases according to

$$
\mathrm{d} n / \mathrm{d} t=-\gamma n
$$

the concentration $N(t)$ of colour centres will be given by

$$
N(t)=n(0)-n(t)=n(0)\left(1-e^{-\gamma t}\right)
$$

what has obviously the same form as eq.(13). It is plausible that indeed latent centres are involved since in general the irradiation colours the mineral in an inhomogeneous but nevertheless reproducible way.

4.3. Supplementary measurements. We also carried out some absorption measurements in the infrared region. In the infrared region down to $400 \mathrm{~cm}^{-1}$ we employed $\mathrm{KBr}$ pellets and for the region between $400 \mathrm{~cm}^{-1}$ and $200 \mathrm{~cm}^{-1}$ CsI pellets.

The spectrum exhibits a broad band around $1000 \mathrm{~cm}^{-1}$ which is considered to be characteristic for alumino-silicates and is attributed ${ }^{16}$ ) to the "stretching" modes of the $\mathrm{AlO}_{4}$ and $\mathrm{SiO}_{4}$ groups. Two bands around $700 \mathrm{~cm}^{-1}$ and $450 \mathrm{~cm}^{-1}$ are presumably more characteristic for the specific sodalite structure ${ }^{17}$ ). Our measurements confirm those of Taylor et al. ${ }^{18}$ ) who have reported the spectrum down to $400 \mathrm{~cm}^{-1}$. Between $400 \mathrm{~cm}^{-1}$ and $200 \mathrm{~cm}^{-1}$ we did not detect new absorption bands.

The described measurements were performed on a sample in its natural condition. The same sample was used for recording a Raman spectrum, however, without finding any line.

4.4. Connection with the dielectric losses. An indication for a connection between the colour centre and the dipole centre will obviously be obtained if somehow a relation exists between the concentration $N_{\mathrm{c}}$ of the colour centres and the concentration $N_{\mathrm{d}}$ of the dipole centres.

Comparison of the maximum values of both concentrations for any sample seems most appropriate. The intended values are not known explicitely, but, in any case, they are proportional to $\alpha_{\mathrm{m}}$ and to $T \tan \delta_{\max }$, respectively. We therefore plotted the maximum value of $T \tan \delta_{\max }$, i.e. its value in the uncoloured state $(t=0)$, versus the maximum value of $\alpha_{\mathrm{m}}, \alpha_{\mathrm{s}}$, in fig. 5 (as in section 3.2 we took the value of the loss in relaxation process I) for several samples.

From fig. 5 we may conclude that a linear relation exists between 


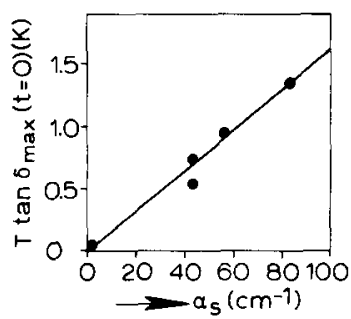

Fig. 5. The experimental relation between the maximum values of the loss and the absorption coefficient for several samples.

$N_{\mathrm{d}}(t=0)$ and $N_{\mathrm{c}}(t=\infty)$, or, in other words, a connection between the dipole centre and the colour centre.

5. ESR experiments. It is a well-known fact that in many cases irradiation-induced defects are also paramagnetic. For this reason we investigated the ESR spectrum of our samples.

Usually we powdered the samples and put them in a quartz ampul in the cavity. We checked that powdering did not influence the spectrum. The sample could be irradiated with ultraviolet light from a high-pressure mercury lamp during the experiments. This possibility was of some importance because, as appears from the work of Hodgson et al $^{6}{ }^{6}$ ), in synthetic sodalites F-centres can be formed under UV irradiation by trapping electrons in halide ion vacancies (if they are present).

Most of the experiments were carried out in the temperature region between room temperature and $77 \mathrm{~K}$.

5.1. Description of the ESR spectrum. The ESR spectrum turned out to be a single line; in fig. 6 we give a typical example for a 5 hour $\mathrm{X}$-irradiated sample, recorded at liquid-nitrogen temperature. Whereas the $\mathrm{X}$ irradiation enhances the line intensity, the UV irradiation did not alter the spectrum at all.

The experimental $g$ value is 2.011 . Being larger than the free electron value $(g=2.0023)$, this value suggests that we are dealing with a hole centre $\left.{ }^{19}\right)$. In any case the line is not caused by the above mentioned F-centre which has $g=2.002$ and exhibits a strong hyperfine splitting due to the four surrounding sodium ions ${ }^{6}$ ).

The $g$ value does not depend on the temperature $T$. The line width turns out to be temperature dependent: it increases from 8.5 gauss at $77 \mathrm{~K}$ to about 12 gauss at room temperature. The line intensity is proportional to $T^{-1}$, obeying the Curie law. Recording the spectrum at about $10 \mathrm{~K}$ did not reveal new information. Especially the line width took the same value as at $77 \mathrm{~K}$. 


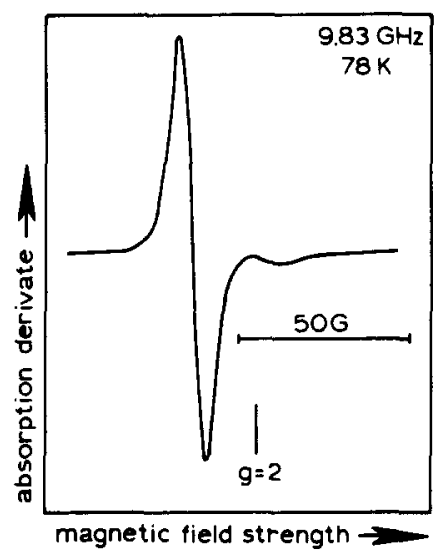

Fig. 6. Ty pical recording of the ESR signal (sample E-4 after 5 hours of $\mathrm{X}$ irradiation).

All these characteristics did not change by $X$ irradiation. Likewise, they did not much depend on the choice of the sample.

Fig. 6 also reveals that at the high-field side of the line a second much weaker signal is present. We could detect the signal, situated at a magnetic field strength corresponding to a $g$ value of 1.997 , in all investigated samples. Since both signals at least qualitatively behave in a similar way under X irradiation one could suppose that they belong to each other. A possible explanation would be that we are dealing with a second centre, its generation by $\mathrm{X}$ irradiation being connected to the generation of the hole centre. On account of the negative $g$ shift one could suppose the centre to be an electron centre.

5.2. The growth curve. We determined the growth curve of the ESR signal by including in the ampul, containing the sodalite specimen, also a charcoal sample* with a known number of spins, acting as a reference sample. By comparing the signals and assuming that the same spin values are involved one can calculate the concentration $N_{\mathrm{S}}$ of $\operatorname{spins}^{20}$ ).

First we observed that the ESR signal vanishes if the sample under investigation is heated up to the same temperature as where the colour bleaches. Assuming that the effect of the irradiation is additive, as we did before, we determined the growth curve by measuring the ESR signal at liquid-nitrogen temperature after every irradiation step. The experimental results for one sample are shown in fig. 7 where we plotted $N_{\mathrm{S}}$ versus the

*Thanks are due to Dr. G. Casteleijn of this laboratory for putting this sample at our disposal. 


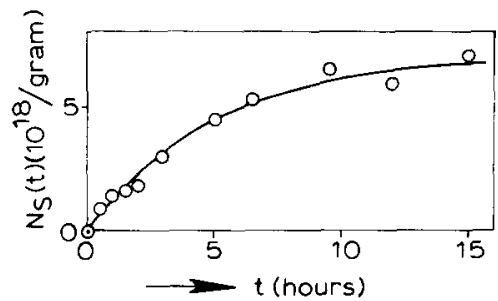

Fig. 7. The experimental growth curve for the spin concentration (sample F-o ${ }^{b}$ ).

irradiation time $t$. We see that the number of spins per gram grows in a similar way as does the absorption coefficient. It can be described analogous to eq. (13) by:

$$
N_{\mathrm{S}}=N_{\mathrm{Ss}}\left[1-\exp \left(-\gamma_{\mathrm{S}} t\right)\right],
$$

where $N_{\mathrm{Ss}}$ denotes the saturation value. For $\gamma_{\mathrm{S}}$ we found for two different samples (for $\mathrm{F}-0^{\mathrm{b}}$ and $\mathrm{E}-4$, respectively):

$$
\gamma_{\mathrm{S}}=0.2 \text { hour }^{-1} \text { and } 0.4 \text { hour }^{-1} \text {. }
$$

The discussion given in section 4.2 of course also applies in this case.

5.3. Connection with the colour centre. There are at least two indications for a close connection between the centre responsible for paramagnetic absorption and the colour centre. First, the fact that both centres disappear at the same temperature (thermal bleaching). Secondly, $\mathrm{X}$ irradiation seems to restore both centres simultaneously and in the same way: besides the fact that the growth curves can be described by equivalent expressions, $\gamma_{c}$ and $\gamma_{S}$ [eqs. (14) and (17)] are of the same order of magnitude.

The connection will become more evident if we compare the spin concentration with the colour centre concentration. Therefore we plotted in fig. $8 N_{\mathrm{Ss}}$ versus $\alpha_{\mathrm{s}}$, which is proportional to $N_{\mathrm{cs}}$, for some samples.

Fig. 8 proves the existence of a linear relationship between the concerning concentrations.

On account of these facts we believe that both centres either are manifestations of the same centre or have a common precursor. Assuming that every spin corresponds to one paramagnetic centre, we can now calculate from the spin concentration $N_{\mathrm{S}}$ and the specific density $\rho\left(=2.3^{15}\right)$ ) the concentration of colour centres $N_{\mathrm{c}}$. In addition we can determine the oscillator strength of the optical transition by using eq. (12). 


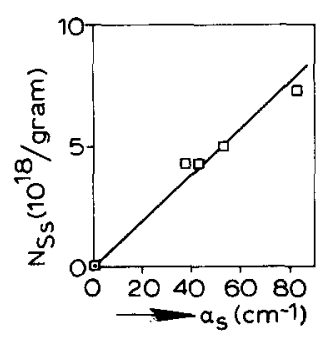

Fig. 8. The experimental relation between the saturation values of the absorption coefficient and the spin concentration for several samples.

We find as a result: $f=2.2 \times 10^{-2}$. From this value we infer that probably no $\mathrm{F}$ centres are involved.

6. Annealing experiments. The colour centre, responsible for the absorption around $610 \mathrm{~nm}$, is stable up to a temperature of about $450^{\circ} \mathrm{C}$. At this temperature the colour vanishes fastly. The dielectric loss, on the other hand, is maximal in these bleached samples. The question arises whether also for the dipole centre a critical temperature or temperature region exists where the centre loses its characteristic features.

For that purpose we heated some samples in a platinum container in air. We avoided heating in vacuo or in a reducing environment because this seems to influence the defect-chemical properties, at least in the case of synthetic sodalites ${ }^{3,6}$ ). Within a measuring accuracy of $0.5 \%$ we did not find any change in the weight of the samples after any heat treatment. After annealing a sample at a temperature $T_{\mathrm{a}}$ during some time $t$ the loss was measured in the temperature region around $100 \mathrm{~K}$ (relaxation process I).

First we observed that prolonged or brief bleaching at $450^{\circ} \mathrm{C}$ and recycling the process of irradiation and bleaching in all cases led to the same value of $T \tan \delta$ of the investigated sample (H-1). Next we raised $T_{\mathrm{a}}$ several times by 50 to 100 degrees. After annealing at $725^{\circ} \mathrm{C}$ during one hour the decrease of the loss was less than $5 \%$ of its value at $450^{\circ} \mathrm{C}$. Not until the sample was annealed at $825^{\circ} \mathrm{C}$ could we observe a clear decrease of the loss. We therefore repeated the annealing a few times at this temperature. In fig. $9 T \tan \delta_{\max }$ is plotted versus the total time of annealing at $825^{\circ} \mathrm{C}$. We see that the loss decreases exponentially and finally reaches a constant value, which appeared to be a definite one: after irradiating during 18 hours and annealing during one hour at $500^{\circ} \mathrm{C}$ we found a same value of $T \tan \delta_{\max }$.

The loss decreases according to $\exp (-\lambda t)$, where the quantity $\lambda=1.65$ $\times 10^{-4} \mathrm{~s}^{-1}$ at $825^{\circ} \mathrm{C}$. We may interpret $\lambda$ as the rate of dipole centre 


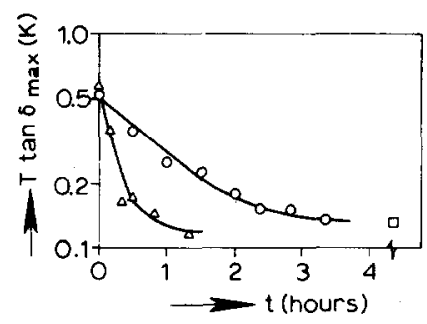

Fig. 9. Decrease of the loss as a function of the total annealing time $t$ for the samples $\mathrm{H}-1\left(\mathrm{O} ; T_{\mathrm{a}}=825^{\circ} \mathrm{C}\right)$ and $\mathrm{H}-3\left(\triangle ; T_{\mathrm{a}}=875^{\circ} \mathrm{C}\right)$. $\square$ denotes the value of the loss after the annealing at $825^{\circ} \mathrm{C}$ followed by 18 hours of $\mathrm{X}$ irradiation and annealing for one hour at $500^{\circ} \mathrm{C}$.

destruction. Expecting $\lambda$ to be a temperature-dependent quantity we repeated the above experiments at a higher value of $T_{\mathrm{a}}$, i.e. at $870^{\circ} \mathrm{C}$. Owing to the irreversibility of the experiment we had to use a neighbouring sample (H-3) instead of $\mathrm{H}-1$. The results were also plotted in fig. 9.

Assuming that both samples are comparable we plotted both values of $\lambda$ together with the estimated value at $725^{\circ} \mathrm{C}$ versus $T_{\mathrm{a}}^{-1}$. The result, fig. 10, suggests the following relation between $\lambda$ and $T_{\mathrm{a}}$ :

$$
\lambda=\lambda_{0} \exp \left(-E / k T_{\mathrm{a}}\right),
$$

where $\lambda_{0} \approx 3 \times 10^{8} \mathrm{~s}^{-1}$ and $E \approx 2.0 \mathrm{eV}$.

The irreversibility of the experiments makes us suppose that one or more of the constituents of the dipole centre during the annealing

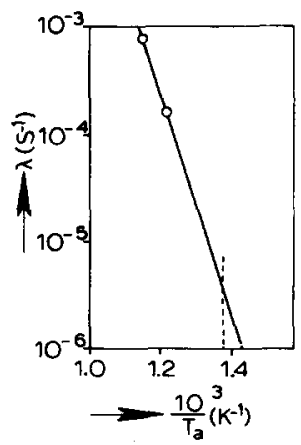

Fig. 10. Plot of the value of $\lambda$ versus $T_{\mathrm{a}}^{-1}$. The broken line indicates the estimated value at $725^{\circ} \mathrm{C}$. 
diffuses through the lattice to a new equilibrium position by which the centre loses is ty pical dipole character. Considering $E$ to be an activation energy one would indeed expect $E$ to be of the order of a few electronvolts.

The final values of the losses are about 20 to $25 \%$ of the initial values at $450^{\circ} \mathrm{C}$. We determined the ESR spectrum of the $\mathrm{H}-3$ sample after prolonged $\mathrm{X}$ irradiation. The intensity of the signal was about a quarter of the value found for the neighbouring, not annealed, sample $\mathrm{H}-2$ like we expected from the results of section 5.3. The line itself had the shape as in fig. 6. Finally we must remark that the colour of $\mathrm{H}-3$, being purple-blue after irradiation, slowly changed into grey-blue by daylight. Apparently some new types of centres were formed during the heat treatments.

7. Discussion. 7.1. Introduction; colouring and bleaching. Experimentally, we distinguished three centres: a dipolar centre, a colour centre and a paramagnetic centre. In the discussion of the experimental results, however, we will assume that the colour centre is also responsible for the ESR signal as is made plausible in section 5.3. The attribution of the two relaxation processes to one dipole centre will be justified later on.

From the linear relation between $\alpha_{\mathrm{s}}$ and the maximum value, i.e. the value after thermal bleaching (irradiation time $t=0$ ), of the loss $T \tan \delta_{\max }$ we infer that the maximum number of colour centres $N_{\mathrm{cs}}(t=\infty)$ equals the maximum number of dipole centres $N_{\mathrm{d}}(t=0)$. We shall proceed on the basis of the hy pothesis that both kinds of centres are defects with deviating valencies. In order to preserve electro-neutrality we shall suppose specifically that to every defect, responsible for the colour centre, there belongs a charge compensating defect to which the existence of the dipole centre can be attributed.

On account of the observed positive $g$ shift we consider the colour centre as having trapped a hole and the dipole centre as having released an electron. We denote these centres by $A_{c}$ and $A_{d}$, respectively, and their precursors by $A_{c}^{*}$ and $A_{d}^{*}$.

The primary result of the X-irradiation is the creation of holes and electrons in valence and conduction bands. In the bleached state, consisting of $A_{d}$ and $A_{c}^{*}$ centres, part of the holes will be captured at sites $A_{c}^{*}$ where upon the centres $A_{c}$ arise. Electrons, captured by the $A_{d}$ centres, convert these into dielectrically inactive centres $A_{d}^{*}$. Part of the electrons are also captured by (other) electron traps $A_{t}$. The presence of these traps, which may vary in kind and concentration from sample to sample, is suggested by the way in which the dielectric loss decreases under $\mathrm{X}$ irradiation (sec. 3.2). Hole traps seem to play a minor part in our model. 
Summarizing, the following reactions occur:

$$
A_{c}^{*}+h \rightarrow A_{c}, \quad A_{d}+e \rightarrow A_{d}^{*}, \quad A_{t}+e \rightarrow A_{t}^{*}
$$

under the condition that $\left[\mathrm{A}_{\mathrm{c}}\right]=\left[\mathrm{A}_{\mathrm{d}}^{*}\right]+\left[\mathrm{A}_{\mathrm{t}}^{*}\right]$, where square brackets denote concentrations.

The reserved process of the colouring, i.e. the bleaching, means that the electron in $A_{d}^{*}$ and the hole in $A_{c}$ recombine, restoring the dipole centre $A_{d}$ and the "inactive" centre $A_{c}^{*}$. The bleaching may start by excitation either of the electron from $A_{d}^{*}$ to the conduction band or of the hole from $A_{c}$ to the valence band. Schematically we get

$$
A_{d}^{*}\left(A_{t}^{*}\right) \rightarrow A_{d}\left(A_{t}\right)+e, \quad A_{c}+e \rightarrow A_{c}^{*}
$$

or

$$
A_{c} \rightarrow A_{c}^{*}+h, \quad A_{d}^{*}\left(A_{t}^{*}\right)+h \rightarrow A_{d}\left(A_{t}\right) .
$$

An objection against the first mechanism is that it is not very likely that $A_{d}^{*}$ and $A_{t}^{*}$ release their electrons at the same temperature, as would be required from the completeness of the bleaching at $450^{\circ} \mathrm{C}$. Therefore, we prefer a bleaching mechanism via the valence band.

The proposed mechanisms of colouring and bleaching are visualized in fig. 11.

7.2. Analysis of the dipole centre. On account of the occurrence of two relaxation processes (labelled I and II, section 3.1) one could suppose the existence of two different dipole centres $A_{d}, e . g$. in the form of two kinds of impurities. One should then explain why the ratio $\eta^{2}$ (section 3.1) is independent of both the irradiation time and the specific sample choice.

We may avoid these difficulties by attributing both relaxation processes to one single defect. As a consequence this defect must be able to

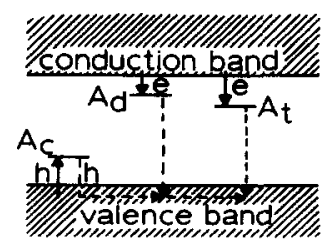

Fig. 11. Comprehensive scheme of the proposed mechanisms for colouring and bleaching (solid and broken arrows, respectively). The differences between $A_{c}$ and $A_{c}^{*}$ centres etc. have not been indicated. 
relax in several ways with different relaxation times. This concept of multiple relaxation is not unusual although not many concrete examples are known. As a matter of fact it has been pointed out, especially by Nowick et al. ${ }^{21}$ ), that many defects should be able to exhibit, at least in principle, multiple relaxations, merely as a consequence of their symmetry properties. In view of these remarks we believe that it is reasonable to assume that in both relaxation processes the same defect is involved.

We describe the dipole centre in terms of a slightly modified version of the well-known Fröhlich model ${ }^{11}$ ). The latter consists of two potential minima $\mathrm{A}$ and $\mathrm{B}$, separated by a barrier of height $U_{\mathrm{I}}$. The jump of a charged particle between both minima corresponds to a roalignment of a dipole with moment $p_{0}$. We modify this model by assuming that both minima in fact consist of two relatively shallow sub-minima $A_{1}$ and $A_{2}$ and $\mathrm{B}_{1}$ and $\mathrm{B}_{2}$, respectively, with corresponding barrier height $U_{\mathrm{II}}$. In fig. 12a we outlined this potential "landscape" as a function of a configuration coordinate $Q$.

At sufficiently low temperatures the motion of the particle will be confined to jumps between $A_{1}$ and $A_{2}$, or between $B_{1}$ and $B_{2}$, whereas at higher temperatures jumping over the barrier $U_{\mathrm{I}}$ becomes increasingly important. We therefore attribute relaxation process I to a motion between the minima $\mathrm{A}$ and $\mathrm{B}$ and process II to a motion within each minimum. The barrier heights can now be interpreted as the activation energies in the expressions for the relaxation times $\tau=\tau_{0} \exp (U / k T)$ [cf. eqs. (3) and (4)]:

$$
U_{\mathrm{I}}=150 \mathrm{meV} \text { and } U_{\mathrm{II}}=55 \mathrm{meV} \text {. }
$$

The jump distances $\mathrm{AB}=L_{\mathrm{I}}$ and $\mathrm{A}_{1} \mathrm{~A}_{2}=\mathrm{B}_{1} \mathrm{~B}_{2}=L_{\mathrm{II}}$ can be calculated if we know the dipole moment $p_{0}$ :

$$
p_{0}=\frac{1}{2} e L \text {. }
$$

a.

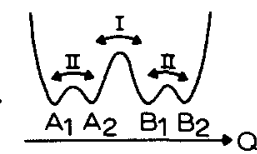

b.

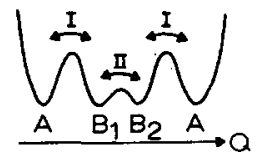

Fig. 12. a. Potential, versus a configuration coordinate, in which a charged particle may perform two kinds of jumping motions.

b. Possible variation on the potential of fig. 12a. 
Including local field corrections in the dipole moment we may determine an "effective" moment $p=\beta p_{0}$, where $\beta$ is a local field correction factor, from the maximum value of the loss [cf. eq.(2)]

$$
\tan \delta_{\max }=\frac{1}{2}(C / T)=2 \pi \chi / s
$$

in which $\chi$ is the susceptibility of the dipole system. Provided that $p F \ll$ $k T$ ( $F$ is the applied field) $\chi$ is given by a Curie law:

$$
\chi=N_{\mathrm{d}} p^{2} / 3 k T \text {. }
$$

The factor $1 / 3$ follows from the assumption that the dipoles are oriented at random in the absence of an external field. Employing the experimental relations between $\alpha_{\mathrm{s}}$ and $N_{\mathrm{Ss}}$ and between $\alpha_{\mathrm{s}}$ and $T \tan \delta_{\max }$ (fig. 8 and fig. 5) and putting $N_{\mathrm{d}}(t=0)=\left[A_{\mathrm{c}}(t=\infty)\right]=N_{\mathrm{Ss}}$ (see section 7.1) we can relate $N_{\mathrm{d}}$ with $T \tan \delta_{\max }$ also experimentally. A typical set of values is: $\alpha_{\mathrm{s}}=50 \mathrm{~cm}^{-1}, N_{\mathrm{d}}(t=0)=1.15 \times 10^{19} \mathrm{~cm}^{-3}$ and $T \tan \delta_{\max }=0.80 \mathrm{~K}$ (process I). From the value of $T \tan \delta_{\max }$ and of $\epsilon_{\mathrm{s}}\left(\approx 8.5^{22}\right)$ ) we may now calculate the susceptibility and consequently the effective dipole moment:

$$
p_{\mathrm{I}}=6.2 \text { debye; } \quad p_{\mathrm{II}}=2.8 \text { debye }
$$

in which $p_{\mathrm{II}}$ was calculated from $p_{\mathrm{I}}$ by using the corrected value for $\eta$ [eq. (6)]. If we may apply a Lorentz field correction $\beta=\left(\epsilon_{\mathrm{s}}+2\right) / 3$ we find for $p_{0}$ :

$$
p_{\text {oI }} \approx 1.8 \text { debye; } \quad p_{\text {oII }} \approx 0.8 \text { debye }
$$

and, by taking for $e$ the electron charge,

$$
L_{\mathrm{I}} \approx 0.75 \AA ; \quad L_{\mathrm{II}} \approx 0.35 \AA .
$$

We finally make a few remarks about this model and its analysis: (i) The potential landscape in fig. 12a is not the only one to which the above analysis is applicable. The model outlined in fig. $12 \mathrm{~b}$ is another possibility, leading to similar results.

(ii) Often occurring combinations of $\tau_{0}$ and $U$ values have been tabulated by Volger and Stevels ${ }^{23}$ ). The combination of $\tau_{0} \approx 10^{-13}$ and $U \approx 0.1 \mathrm{eV}$ as we found seems to be rather characteristic for the case of an ion performing restricted movements. Moreover, one would not expect in that case large dipole moments. Our experimental values agree with this. In a 
crude approximation one could say that the jump distances $L_{\mathrm{I}}$ and $L_{\mathrm{II}}$ are more of the order of ionic radii rather than of the order of interatomic distances in a crystal lattice. This justifies indeed the concept of restricted movements.

Summarizing we may visualize the dipole centre $A_{d}$ as an ion performing rather localized wobbling motions.

7.3. Defects in the sodalite structure. We will try to determine the chemical nature of the centres of which we mentioned above a number of physical properties.

In view of the sodalite structure we may, at least roughly, distinguish: a. Defects in the sodalite cages. This group of defects has received some attention owing to the photochromic and cathodochromic properties of sodalite. The presence of $\mathrm{F}$ centres and of sulfur or oxygen containing complexes, like $\mathrm{S}^{2-}, \mathrm{SO}_{4}^{-}$and $\mathrm{O}_{2}^{-}$, has been demonstrated by ESR or luminescence experiments $\left.{ }^{6-8,24-26}\right)$. However, although in particular sulfur is a rather common impurity in sodalite, we cannot identify the colour centre $A_{c}$ with any of the above mentioned contaminations in view of our optical and ESR experiments. In particular it is ruled out that the $S_{3}^{-}$molecule which shows an absorption band right at $610 \mathrm{~nm}^{27}$ ), would be responsible for the blue colour of sodalite. This conclusion is confirmed by spectrochemical analysis since in most of investigated samples no sulfur could be detected. The only impurity which was present to an amount comparable with the colour centre concentrations, is iron ( 0.01 to 0.1 weight percent). There was, however, no correlation between the iron concentration and the $A_{c}$ centre concentration. We believe therefore that there is no direct connection between the colour centre and an iron impurity.

b. Defects in the sodalite framework. It is a well-known fact, in particular from the investigations in silicate glasses, that numerous defects may exist in such networks. An enumeration and classification of the most important possibilities in silicon oxygen networks has been given by Stevels and $\mathrm{Kats}^{28}$ ). Several defects may be combined into defect "complexes" with complicated structures. As more "elemental" point defects could be mentioned ${ }^{28}$ ):

(i) silicon and aluminium vacancies and silicons or aluminiums at interstitial sites. Their occurrence is not very likely in view of their tendency to form networks.

(ii) oxygen vacancies and interstitial oxygen. Starting from a bridging oxygen ion (i.e. shared by two adjacent oxygen tetrahedra), two kinds of interstitials may be distinguished: the nonbridging oxygen ion which is connected via one bond only with the framework, and the oxygen which is 
completely free from the network. These defects are of some importance as it is believed that especially the oxygen ions are attacked by $\mathrm{X}$ rays, resulting in the loss of an electron and/or their displacement into an interstitial position ${ }^{29}$ ).

(iii) substitutional replacement of silicon or aluminium by other cations like $\mathrm{Ge}^{4+}, \mathrm{B}^{3+}$, etc. A very common defect is an $\mathrm{Al}^{3+}$ at a $\mathrm{Si}^{4+}$ site. When the replacing cation has a deviating charge, charge compensating ions may be present, often in interstitial positions.

The chemical analysis did not reveal an obvious relation between the impurities and the dipole and colour centres. Apparently we are dealing with defects in or closely related to the alumino-silicate framework.

7.4. Proposal for a model. Our starting point is that the dipole centre and the colour centre are connected by the mechanism of charge compensation (see section 7.1). A very simple way to realize such a situation is the replacement of a $\mathrm{Si}^{4+}$ ion by an $\mathrm{Al}^{3+}$ ion in a substitutional way. A monovalent positive ion, say a $\mathrm{Na}^{+}$, at an interstitial site will be assumed to act as charge compensator. We outlined this situation in a very schematic way in fig. 13a. As a matter of fact this is a well-known situation in silicates.

We assume that, under the influence of $\mathrm{X}$ irradiation, one of the oxygens, surrounding the $\mathrm{Al}^{3+}$ ion, loses an electron. This electron, leaving behind a hole, may be captured by the $\mathrm{Na}^{+}$ion.

We can imagine that the hole created in the sodalite framework remains trapped on the oxygen ligands around the $\mathrm{Al}^{3+}$ ion (fig. 13b). Such a centre has been observed in quartz ${ }^{30}$ ) and in alumino-silicate glasses ${ }^{31}$ ) by means of ESR techniques. Another possibility would be that the hole is trapped at one specific oxygen showing, however, super-hyperfine interaction with two adjacent aluminium ions (fig. 13c). Bershchov et al. ${ }^{24}$ ) have reported the ESR spectrum of such a centre in a sodalite single crystal.

These two possibilities do not appear to account for the experimental results. Therefore, we suppose as a third possibility that, in addition, the oxygen which lost one or more electrons is displaced upon irradiation and becomes an interstitial and nonbridging ion (fig. 13d).

We discuss now the relations between these defects and the observed dielectrical, optical and ESR phenomena.

1) The $A_{d}$ centre. The $\mathrm{Na}^{+}$ion, wobbling at its interstitial site between several adjacent equilibrium positions (assumed that these are indeed present), in our opinion is responsible for the occurrence of the dielectric losses (see section 7.2). Fig. 13a therefore refers to the bleached state: an $A_{d}$ centre and an $A_{c}^{*}$ centre. The electron, released from an oxygen 


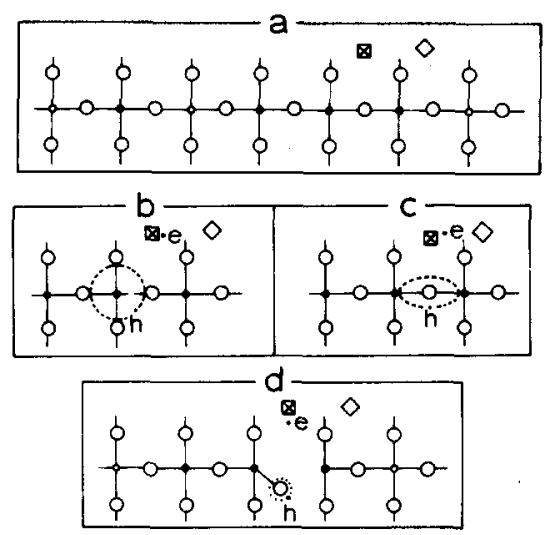

Fig. 13. Strongly simplified scheme of the alumino-silicate network in sodalite; o and - denote silicon and aluminium ions, respectively, and $\bigcirc$ oxygen ions. (Note that actually the $\mathrm{Si}-\mathrm{O}-\mathrm{Si}$ angle in silicates may deviate considerably from $180^{\circ}$ ).

a. $\mathrm{A} \mathrm{Si}^{4+}$ ion has been replaced by an $\mathrm{Al}^{3+}$ ion, accompanied by an extra interstitial monovalent ion, e.g. a sodium ion $(\otimes)$. The presence of electron traps has been indicated by $\diamond$. This situation refers to the bleached state.

$\mathrm{b}$ and $\mathrm{c}$. Two models for an irrddiated state where a hole $(\mathrm{h})$ has been trapped by -schematically - the $\mathrm{AlO}_{4}$ tetrahedron or by an oxygen shared by two tetrahedra and an electron (e) by the $\mathrm{Na}^{+}$ion.

d. Scheme of the proposed model of the irradiated state, consisting of a nonbridging oxygen ion on which a hole has been trapped and a sodium ion or another electron trap which has captured an electron.

upon irradiation, may be trapped on the $\mathrm{Na}^{+}$ion, resulting in the disappearance of the dipole character of this ion at its interstitial site and consequently of its contribution to the dielectric loss: the $A_{d}$ centre is converted into an $A_{d}^{*}$ centre. (We do not exclude that the electron may be captured by another trap. This matter was already discussed in section 7.1.) Simultaneously the $A_{c}^{*}$ centre is converted into the $A_{c}$ centre.

2) The $A_{c}$ centre gives rise to the ESR signal. We assume that it concerns an unpaired hole, trapped on the nonbridging oxygen ion. This assignment is based on the fact that Van Wieringen and Kats ${ }^{32}$ ) observed a resonance in an alumino-silicate glass at the same $g$-value of 2.011 , which they attributed to such a centre. Lee and Bray ${ }^{31}$ ) and Sidorov and Tyul'kin ${ }^{33}$ ), studying similar glasses, arrived at the same conclusion. They found an asymmetric line as we did; however, the linewidth in these glasses (about 20 gauss) and the asymmetry are substantially larger than in our case and temperature independent (we found about 8 gauss and 12 gauss at $78 \mathrm{~K}$ and $300 \mathrm{~K}$, respectively). That we did not detect a hyperfine spitting due to the aluminium nucleus, is consistent with the opinion of Sidorov and Tyul'kin $^{33}$ ) that the hole must be strongly localized at the oxygen. 
In view of the close relationship between the optical and paramagnetic behaviour (section 5.3) we attribute the optical absorption at $2.0 \mathrm{eV}$ to the optical excitation of the hole. The observed asymmetry in the absorption line (see section 4.1) is perhaps related to the existence of a second transition at a photon energy of about $2.7 \mathrm{eV}$. We note that the simultaneous occurrence of two bands at these energies in silicate glasses has been reported several times ${ }^{34}$ ). A relation between these bands and an ESR signal with $g=2.01$ has been established by Sidorov and Tyul'kin ${ }^{35}$ ). These authors studied the optical absorption and ESR spectra of irradiated alkali-silicate glasses. In some cases, depending on the concentration and type of the alkali metal, the ESR line was found to consist of two components, with which the optical absorption bands could be related. On account of these facts they attributed the absorption both near $2 \mathrm{eV}$ and $2.7 \mathrm{eV}$ to a hole trapped on a nonbridging oxygen; the band near $2.7 \mathrm{eV}$ arises if this hole centre is disturbed by an alkali ion in the immediate vicinity. We believe that these data support our interpretation of the optical absorption.

In section 7.1 we concluded that the bleaching mechanism apparently starts at the $A_{c}$ centre. This implies that the nonbridging oxygen moves in such a position that the recombination of the electron and the hole is possible. Presumably the broken $\mathrm{Al}-\mathrm{O}$ band is restored. During this process intermediate states as outlined in figs. $13 \mathrm{~b}$ and $13 \mathrm{c}$ could occur, which apparently are not stable, at least not at the temperature required for the bleaching $\left(450^{\circ} \mathrm{C}\right)$. It is known, indeed, that the centre of fig. $13 \mathrm{~b}$ does not exist above $350^{\circ} \mathrm{C}$, nor in quartz, nor in glasses ${ }^{31}$ ).

The creation of lattice defects by means of $\mathrm{X}$ rays, or in some cases even by UV quanta, is a well-known phenomenon. Yet the underlying mechanisms of displacement and especially of stabilization of the created situation, are understood mainly in a qualitative way and in some specific cases only ${ }^{36}$ ). In particular the mechanism preventing the immediate recombination of interstitial and vacancy is complicated. Perhaps strain fields, due to dislocations, may play an important part as has been pointed out by Seitz ${ }^{37}$ ).

In section 6 we described how annealing of the samples above $750^{\circ} \mathrm{C}$ results in a strong irreversible decrease of the loss. We propose as a possible explanation that after the annealing the alkali ion occupies another position in the lattice where it is not able to perform the restricted movements responsible for the occurrence of the losses. The properties of the $A_{c}$ centre also changed by such an annealing as follows from the observed decrease of the ESR signal. It is not obvious what exactly happens; maybe the $A_{c}$ centre cannot be formed when the distance between the $\mathrm{Na}^{+}$and $\mathrm{Al}^{3+}$ becomes large. 
Besides the spectrum of the hole we should also expect in the irradiated state an ESR spectrum from the electron, released from the $A_{c}^{*}$ centre and captured by the $\mathrm{Na}^{+}$ion or another trap. Van Wieringen and Kats ${ }^{32}$ ) have indeed found a broad line with $g \approx 1.97$ in silicate glasses, which they ascribed to such a trapped electron. The spectrum, however, appears not always to be detectable; Griffiths et al. ${ }^{30}$ ) for instance did not observe it in their studies of irriated quartz. We already noted (section 5.1) that the signal at the high-field side of the main ESR signal could be due to another centre than the $A_{c}$ centre: maybe it is connected with the trapped electron.

\subsection{Final remarks. With the help of this tentative model we were} able to explain at least the main features of the centres, responsible for the dielectric, optical and paramagnetic effects, and their mutual relations.

The choice of an $\mathrm{Al}^{3+}$ ion as the substitutional ion and of a $\mathrm{Na}^{+}$ion as charge compensator is supported by a similar case of an $\mathrm{AlO}_{4}-\mathrm{Na}$ defect, as outlined in fig. 13a, in unirradiated quartz, studied by means of dielectric relaxation experiments. The authors ${ }^{38}$ )suggested a "potential landscape" in which the $\mathrm{Na}^{+}$ion moves (fig. 14), similar to what was proposed in section 7.2 (The agreement between several parameters is even striking.) Their model was, moreover, confirmed by Nowick and Stanley ${ }^{39}$ ), using a group theoretical analysis.

If this resemblence indeed implies the occurrence of the $\mathrm{AlO}_{4}-\mathrm{Na}$ complex in both materials, the intriguing question remains why the influence of the $\mathrm{X}$ rays on the defect in sodalite differs so much from that on the same defect in quartz. In the latter case a hole is trapped by

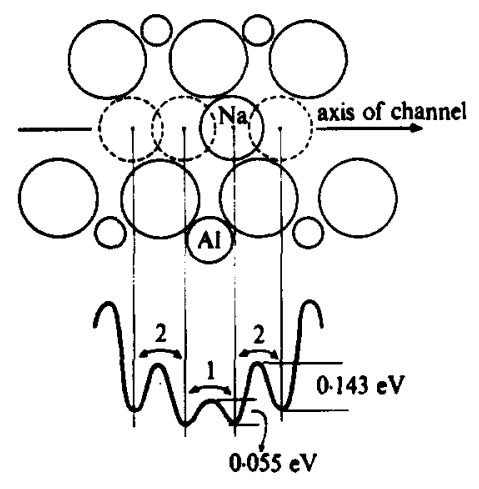

Fig. 14. Model for the $\mathrm{Al}-\mathrm{Na}$ defect in quartz; shown are the equilibrium positions of the $\mathrm{Na}^{+}$ion and the corresponding potential energies. After ref. 38 . 
the $\mathrm{AlO}_{4}$ tetrahedron, the hopping or tunneling motion of the hole between two adjacent oxygens giving rise to dielectric relaxation losses ${ }^{40}$ ). Regarding the $\mathrm{AlO}_{4}$ tetrahedron, by which the hole may be trapped, as an "isolated" structural unit one would expect this hole centre to be present in many other silicates. However, like in sodalite, we failed to find (by means of dielectric loss experiments) its presence in spodumen ${ }^{41}$ ), $\mathrm{LiAl}\left(\mathrm{SiO}_{3}\right)_{2}$, and in berlinite ${ }^{41}$ ), $\mathrm{AlPO}_{4}$ (which exhibits a structure quite similar to $\alpha$-quartz).

An important difference between quartz and these compounds is of course their structure; a second difference which could play a part is that in the former case the cation sub-lattice is uniformly charged, whereas in the other cases the charge of this sub-lattice varies locally. Assuming the $\mathrm{AlO}_{4}$ complex to be rather sensitive for its surroundings (at least as far as it concerns its capability for hole trapping), such a hole centre can apparently only be created when the complex is in a specific highly symmetric environment. This could perhaps explain the occurrence of this hole centre specifically in quartz. This tentative explanation is supported by the absence of relaxation losses, due to the hole, in irradiated silicate glasses, contaminated with aluminium.

Acknowledgement. We wish to thank Professor Dr. H. W. de Wijn for reading the manuscript. The investigations were performed as part of the research programme of the "Stichting voor Fundamenteel Onderzoek der Materie" (F.O.M.) with financial support from the "Nederlandse Organisatie voor Zuiver Wetenschappelijk Onderzoek" (Z.W.O.).

\section{REFERENCES}

1) E.g. Medved, D. B., Am. Mineralogist 39 (1954) 615.

2) Kirk, R. D., Am. Mineralogist 40 (1955) 22; J. Electrochem. Soc. 101 (1954) 461.

3) E.g. Taylor, M. J., Marshall, D. J., Forrester, P. A. and McLaughlan, S. D., Radio Electron. Eng. 40 (1970) July.

4) E.g. Duncan, R. C., Faughnan, B. W. and Philips, W., Appl. Opt. 9 (1970) 2236. Faughnan, B. W., Gorog, I., Heyman, P. M. and Shidlovsky, I., Proc. IEEE 61 (1973) 927.

5) Pauling, L., Proc. Natl. Acad. Sci. 16 (1930) 453.

6) Hodgson, W. G., Brinen, J. S. and Williams, E. F., J. Chem. Phys. 47 (1967) 3719.

7) Schulman, J. H. and Kirk, R. D., Solid State Commun. 2 (1964) 105.

8) McLaughlan, S. D. and Marshall, D. J., J. Phys. Chem. 74 (1970) 1359.

9) Casanova Alig, R., J. Phys. Chem. Solids 35 (1974) 53.

10) Bye, K. L. and White, E. A. D., J. Cryst. Growth 6 (1970) 355.

11) Fröhlich, H., Theory of Dielectrics, University Press (Oxford, 1958). Daniel, Vera, Dielectric Relaxation, Academic Press (London, 1967). 
12) De Vos, W. J. and Volger, J., Trans. Far. Soc. 67 (1971) 1626.

13) Markham, J. J., Solid State Physics, suppl. 8 F. Seitz and D. Turnbull, eds., Academic Press (London, 1966).

14) E.g. Dexter, D. L., Phys. Rev. 101 (1956) 48.

15) Handbook of Chemistry and Physics, Chem. Rubber Co. (Cleveland).

16) Milkey, R. G., Am. Mineralogist 45 (1960) 990.

17) Vierne, R. and Brunel, R., Bull. Soc. fr. Minéral. Cristallogr. 92 (1969) 409.

18) Taylor, M. J., Marshall, D. J. and Evans, H., J. Phys. Chem. Solids 32 (1971) 2021.

19) E.g. Slichter, C. P., Principles of Magnetic Resonance, Harper and Row (New York, 1963).

20) E.g. Poole, C. P., Electron Spin Resonance, Interscience Publisher (New York, 1967).

21) Nowick, A. S. and Heller, W. R., Adv. Phys. 14 (1965) 101. Nowick, A. S., Adv. Phys. 16 (1967) 1.

22) Shaldin, Y. V., Mel'Nikov, O. K., Nabatov, V. V. and Pisarevskii, Y. V., Sov. Phys. Crystallogr. 10 (1966) 484.

23) Volger, J. and Stevels, J. M., Philips Res. Rep. 11 (1956) 452.

24) Bershchov, L. V., Martirosyan, V. O., Platonov, A. N. and Tarashchan, A. N., Neorganisch. Mater. 5 (1969) 1780.

25) Ballentyne, D. W. G. and Bye, K. L., J. Phys. D 3 (1970) 1438.

26) Van Doorn, C. Z., Schipper, D. J. and Bolwijn, P. T., J. Electrochem. Soc. 119 (1972) 85.

27) Morton, J. R., Colloque Ampère XV, North-Holland (Amsterdam, 1969) p.299.

28) Stevels, J. M. and Kats, A., Philips Res. Rep. 11 (1956) 103.

29) Kats, A. and Stevels, J. M., Philips Res. Rep. 11 (1956) 115.

30) Griffiths, J. H. E., Owen, J. and Ward, J. M., Nature 173 (1954) 439.

31) Lee, S. and Bray, P. J., Phys. Chem. Glasses 3 (1962) 37.

32) Van Wieringen, J. S. and Kats, A., Philips Res. Rep. 12 (1957) 432.

33) Sidorov, T. A. and Tyul'kin, V. A., DAN SSSR 175 (1967) 872.

34) E.g. Mackey, J. H., Smith, H. L. and Halperin, A., J. Phys. Chem. Solids 27 (1966) 1759.

35) Sidorov, T. A. and Tyul'kin, V. A., Theor. and Exp. Chem. (U.S.A.) 4 (1968) 61.

36) See for instance Varley, J. H. O., J. Nuclear Energy 1 (1954) 130.

Pooley, D., Brit. J. Appl. Phys. 17 (1966) 855. With respect to the silicates: see also the recent work of Revesz, A. G., e.g. Solid State Commun. 10 (1972) 127.

37) Seitz, F., Rev, Mod. Physics 26 (1954) 7.

38) Stevels, J. M. and Volger, J., Phys. Chem. Glasses 4 (1963) 247.

39) Nowick, A. S. and Stanley, M. W., J. Appl. Phys. 40 (1970) 4995.

40) De Vos, W. J. and Volger, J., Physica 47 (1970) 13.

41) E.g. Hiller, J. E., Grundriss der Kristallchemie (Berlin, 1952). 